\title{
Record Solar Activity in August 1972
}

DURING early August 1972, sunspot activity was responsible for a remarkable series of effects, which were observable on the solar surface, in interplanetary space and at the Earth. Three communications in this issue of Nature (pages 331, 333 and 335) are concerned with these events. Two are devoted to cosmic-ray studies and the third marks the advent of a new branch of solar spectroscopy at ultra-high energies.

Intense solar activity is usually associated with complex sunspot groups which in turn represent regions of enhanced solar magnetic field strength, typically several thousand gauss. Within the sunspot group a solar flare may occur; this is characterized by bursts of X-rays, optical line emission and radio waves at frequencies of the order of hundreds of $\mathrm{MHz}$. Strong solar flares may emit white light for a short period and may also accelerate charged electrons and nuclei to relativistic energies, in the case of protons up to several times $10^{9}$ $\mathrm{eV}$. The nature of the acceleration process of these solar "cosmic" rays is not understood. Perhaps they are pre-accelerated in some large-scale magnetic trap by interaction with magnetic waves and only released during the flare. Alternatively the flare may reflect particle acceleration in a region where oppositely directed mag. netic field lines annihilate each other and an intense electric field is set up.

More detailed angular and wavelength resolution in observations of solar activity is required to help sort out this problem, and the communication by Chupp and his colleagues (page 333) provides suitable new information on the fast protons, previously only observable when they reached the Earth or a space vehicle. Direct knowledge of the accelerated electrons comes from the radio measurements but, in the case of the protons, interaction with matter must be detected. Chupp et al. used a large scintillator crystal on an OSO satellite and on August 4 and 7,1972, recorded spectral emission lines at low energy gamma-ray energies during two flares. Although preliminary measurements in this energy range date as far back as 1958 (Winckler and Peterson, Nature, 181, 1317), this was the first occasion on which line emission has been detected. The lines originate from the interaction of fast protons moving downwards with matter in the atmosphere of the solar photosphere. Secondary positrons are probably responsible for a spectral line seen at $0.51 \mathrm{MeV}$, and deuterons, arising from secondary neutrons, are likely to have caused the 2.22 MeV line found by Chupp et al.

A companion article, by Mathews and Lanzerotti (page 335 ), is related to the previous work. These authors have studied the arrival at the Earth of the accelerated solar "cosmic" rays from the flare on August 7, as measured by some standard monitoring devices. By careful timing they have shown that the energetic protons must have been accelerated at the time of peak flux in the white light emission, rather than at the later time of the peak of radio and optical line emission. Thus it is thought that the white light is caused by heating of the photospheric atmosphere a few hundred kilometres above the solar surface by the downward-moving energetic protons which appeared in the acceleration process (see Svestka, Solar Phys., 13, 471 ; 1970).

A fast stream of plasma is ejected into interplanetary space in association with the flare activity, and it is such a stream which caused the cosmic-ray storm discussed by Pomerantz and Duggal (page 331). Interplanetary space is always filled by the expanding plasma layers of the outer solar corona and this plasma motion, at about $400 \mathrm{~km} \mathrm{~s}^{-1}$, is known as the solar wind. Magnetic field is carried out from the Sun by the highly conducting plasma and is responsible for screening off some relativistic cosmic-ray nuclei from the Galaxy. The charged cosmic rays diffuse inwards, colliding with magnetic scattering centres moving outwards and losing energy as they go. The effect of the fast plasma stream linked with the flare is, however, not simply to enhance the screening process. Observations such as those of Pomerantz and Duggal show that cosmic-ray storms are made up of a series of sharp (Forbush) superimposed decreases in the cosmic-ray intensity at the Earth, often followed by a rapid recovery. Changes of intensity at the rate of 5 per cent an hour were seen in one event. And changes of particle flux are often highly dependent on direction of view. Thus it is logical to look for some large-scale barrier in space as the basis of a way to explain cosmic-ray storms.

The fast stream late on August 4, caused perhaps by a flare on August 2, reached a plasma velocity of 1,100 $\mathrm{km} \mathrm{s} \mathrm{s}^{-1}$, about the highest ever recorded by a Pioneer spacecraft. Preceding the stream was a shock front in which the magnetic field strength jumped from 30 to 40 gamma to 50 to 80 gamma ( 1 gamma $=10^{-5}$ gauss) according to preliminary HEOS-2 satellite data. (At quiet times the strength of the interplanetary field is no more than 6 gamma.) The shock was still very noticeable at the Pioneer on its way to Jupiter, 3.5 AU from the Sun. At the time these plasma changes were taking place the cosmic-ray intensity near the Earth is deduced to have fallen to half of its value before the storm. Only one other comparable event has been recorded, in July 1959. A theoretical explanation of the sharp cosmic-ray decreases can probably be sought either in the barrier associated with the shock jump in interplanetary field strength or, perhaps more likely, in a far-reaching plasma/field discontinuity known as a tangential discontinuity, which is expected to exist within all such interactions between the fast streams and solar wind. Magnetic field lines do not cross a tangential discontinuity, so charged particles should find it an effective barrier. Further joint analysis of satellite, space probe and cosmic-ray data for these events of August 1972 should enable a better picture of the properties of fast plasma streams ejected under flare conditions to be built up.-J. J. Q. 